\title{
Computational Game Theory
}

\author{
Bruno Codenotti \\ Istituto di Informatica e Telematica, \\ Consiglio Nazionale delle Ricerche, \\ Pisa, Italy \\ bruno.codenotti@iit.cnr.it
}

\begin{abstract}
We first provide a quick background in Game and Economic Theory, and then discuss some fundamental computational questions arising in these areas. We will focus on the interplay between Game Theory and Computer Science, with an emphasis on some of the most challenging open questions.
\end{abstract}

